PALABRAS CLAVE

Desarrollo económico

Desarrollo local

Estrategias del desarrollo

Economía regional

Bienes públicos

Capital social

Descentralización gubernamental

Países en desarrollo

Mario D. Tello

Profesor e Investigador Asociado, CENTRUM Católica y Departamento de Economía, Pontificia Universidad Católica del Perú

mtello@pucp.edu.pe
REVISTA CEPAL 102 DICIEMBRE 2010

\section{Del desarrollo económico nacional al desarrollo local: aspectos teóricos}

\author{
Mario D. Tello
}

$\mathbf{S}$ obre la base de una breve y selectiva revisión de la literatura acerca del desarrollo económico local (DEL), en el presente artículo se analizan cuatro aspectos teóricos que distinguen a las teorías de desarrollo económico "local" de las teorías "nacionales". Estos aspectos son: los factores de localización; los bienes y servicios públicos locales: la participación activa de distintos agentes privados, y el enfoque multidisciplinario de las teorías de DEL. Este análisis podría utilizarse para diseñar una disciplina académica de DEL habitualmente ausente en los países en desarrollo y arrojar luces sobre los objetivos y el papel de los agentes en los procesos de descentralización y DEL que actualmente tienen lugar en estos países. 


\section{I}

\section{Introducción}

Las teorías de desarrollo económico local (DEL) y las teorías de desarrollo económico nacional (DEN) son disciplinas muy arraigadas que se enseñan en la mayoría de las universidades de los países industrializados (Blakely, 2003). Aunque los países en desarrollo, en particular los de América Latina, tienen una larga tradición en la puesta en práctica de políticas regionales -como la planificación de cuencas fluviales, los incentivos fiscales, las agencias de desarrollo regional, los polos de crecimiento y el desarrollo integrado rural-que, a principios de la década de 1960, generaron la primera oleada de cursos de posgrado en DEL, solo recientemente (a inicios de la década de 2000) las universidades latinoamericanas volvieron a interesarse por este campo. Los procesos de descentralización promovidos en muchos países en desarrollo a lo largo de las dos últimas décadas (Montero y Samuels, 2004; Stren y otros, 2002; Aghón, Alburquerque y Cortés, 2001; Oxhorn, Tulchin y Selee, 2004; Rondinelli y Cheema, 1983) han contribuido a renovar el interés por los temas relativos al DEL. También las instituciones de desarrollo internacional prestan ahora atención al DEL y lo financian, como puede verse en sus respectivas páginas de Internet. ${ }^{1}$ En general, los aspectos del desarrollo económico local en áreas geográficas (regiones, condados, provincias o departamentos) de los países en desarrollo se analizan mediante el marco de desarrollo económico nacional (DEN), sin incluir las características propias del desarrollo local. Además, los organismos gubernamentales que operan a nivel local y los agentes económicos, sociales y políticos residentes en áreas específicas perciben que el desarrollo

El autor agradece a Esteban Hnyilicza y a un árbitro anónimo por sus útiles comentarios $\mathrm{y}$, en especial, por haberle señalado las primeras aplicaciones de las políticas de desarrollo económico local en los países latinoamericanos.

${ }^{1}$ Por ejemplo, el Banco Mundial, la Comisión Económica para América Latina y el Caribe (CEPAL), las Naciones Unidas, el Banco Interamericano de Desarrollo (BID), la Organización de Cooperación y Desarrollo Económicos (OCDE) y otros. económico de esas áreas depende en gran medida de las intervenciones y las políticas económicas del gobierno central (o federal). ${ }^{2}$ En el presente artículo se intenta tender un puente entre las actuales teorías de DEL y DEN, estudiando brevemente cuatro aspectos teóricos que distinguen a las primeras de las segundas.

El primer aspecto se refiere a los factores de localización vinculados a áreas geográficas específicas dentro de una economía. En la mayoría de las teorías de DEN se supone implícitamente que el territorio de una economía es homogéneo. El segundo aspecto es la naturaleza de los bienes y servicios que suministran diferentes niveles del gobierno. En las teorías de DEN se da por sentado que la influencia de los bienes y servicios públicos suministrados por el gobierno abarca todas las áreas geográficas de una economía. El tercer aspecto es el papel y la participación de los agentes específicos residentes en las áreas locales. En las teorías tradicionales de DEN no se incorpora explícitamente el papel del agente. No obstante, durante las dos últimas décadas en las modernas teorías del DEN se ha atribuido un papel más importante al comportamiento de los agentes, aunque no necesariamente vinculado a las áreas locales en que viven. La cuarta cuestión es el enfoque multidisciplinario del análisis de los temas de DEL. Las teorías de DEN se centran en los fundamentos económicos y en los mecanismos de los mercados, las instituciones y las organizaciones, independientemente de las áreas geográficas en que operan. En las secciones III a VI de este artículo se presenta un estudio breve y selectivo de estos cuatro aspectos. Sin embargo y a manera de punto de partida, en la sección II se abordan algunas de las definiciones de DEL encontradas en la literatura. En la sección VII se ofrecen las conclusiones del trabajo.

\footnotetext{
${ }^{2}$ Véase el estudio de dos regiones del Perú (Tello, 2008).
} 


\section{II}

\section{Definiciones de desarrollo económico local (DEL)}

En la página web del Banco Mundial se sostiene que el desarrollo económico local (DEL) proporciona al gobierno local, los sectores privados, los organismos no gubernamentales y las comunidades locales la oportunidad de trabajar mancomunadamente para mejorar la economía local. El DEL incide en el mejoramiento de la competitividad, aumenta el desarrollo sostenible y asegura la inclusividad del crecimiento por medio de un conjunto de disciplinas, incluidos el planeamiento físico, la economía y el marketing. Asimismo, incorpora numerosas funciones del gobierno local y del sector privado, tales como la planificación medioambiental, el desarrollo de empresas, la provisión de infraestructuras, el desarrollo inmobiliario y la financiación.

En varias contribuciones de la Comisión Económica para América Latina y el Caribe (CEPAL), tales como Aghón, Alburquerque y Cortés (2001) y Finot (2001); y del Banco Interamericano de Desarrollo (BID), como Alburquerque, Llorens y Del Castillo (2002) y Llisterri (2000), el DEL se define como el proceso estructural y de crecimiento que, mediante el máximo aprovechamiento de los recursos locales, permite que las personas que viven en un área local o una región de un país experimenten un incremento continuo de su bienestar. El proceso comprende tres dimensiones: la económica (que incluye los medios de producción por cuyo intermedio las empresas locales pueden usar eficazmente los recursos locales, generar economías de escala y acrecentar su productividad y competitividad en el mercado); la sociocultural (es decir, la red social y económica en que los valores y las instituciones apoyan el proceso de DEL), y la dimensión política y administrativa (que atañe a las iniciativas que crean un entorno local y comercial favorable al fomento del desarrollo económico local).

En la literatura económica y desde la perspectiva de una economía industrializada (Bartik, 1995 y Malizia, 1985), el DEL se ha definido tradicionalmente como aquellos cambios en la capacidad de una economía local que permiten incrementar el crecimiento económico, generar empleo y crear nueva riqueza para los residentes locales. Una definición moderna se encuentra en Blakely (2003) y Blakely y Bradshaw (2002), quienes sostienen que el campo de DEL es una combinación de disciplinas y una amalgama de políticas y prácticas. Hoy los conocimientos de DEL constituyen en sí una pequeña industria en crecimiento. El concepto de DEL se basa en cuatro factores: i) recursos autóctonos y control local; ii) formación de nueva riqueza; iii) desarrollo de nuevas capacidades, y iv) expansión de los recursos.

En las citadas definiciones de DEL se comparten varios aspectos que no suelen tomarse explícitamente en cuenta en las definiciones del desarrollo económico a nivel nacional. El primero de ellos atañe a la localización geográfica del proceso de DEL. Desde el punto de vista geográfico, político o administrativo, los países suelen dividirse (OCDE, 2002) en distintos niveles territoriales o unidades espaciales (por ejemplo: estados, regiones, departamentos, provincias, distritos, municipios y otros), y los residentes (los agentes económicos, políticos y sociales) se identifican con dichos territorios. Greffe (2004) postula al menos tres justificaciones para aplicar el enfoque local al proceso de desarrollo económico: la primera de ellas atiende a las características específicas de ciertos territorios, lo que puede afectar al funcionamiento adecuado de los mecanismos espontáneos del mercado o a las políticas formuladas para un área sin tener en cuenta las características locales. Otra justificación remite a la naturaleza multidimensional de los problemas de empleo, presentados normalmente como un desequilibrio entre la oferta y la demanda. Así, factores como la capacitación, la vivienda o la movilidad, la asistencia médica, las limitaciones del salario mínimo y otros, de hecho solo pueden identificarse y manejarse con precisión y cerca de los interesados, lo que implica que las iniciativas deben planificarse, ejecutarse y coordinarse a nivel local. La última justificación se relaciona con el contexto de economía global de los países, en virtud del cual se justifica el enfoque local, pues permite una mayor sinergia entre las bases económicas y sociales de los mercados.

El segundo aspecto de los procesos de DEL se vincula a la provisión de bienes y servicios públicos a nivel local. ${ }^{3}$ Los gobiernos emplean una serie de

\footnotetext{
${ }^{3}$ McGuire y otros (1994) conceptualizan la capacidad de desarrollo local en función de tres factores generales: la participación ciudadana, la estructura comunitaria o local y los instrumentos de desarrollo. Estos últimos se relacionan con las políticas gubernamentales locales y la provisión de bienes y servicios que apoyan al proceso de DEL.
} 
instrumentos para alcanzar los objetivos de eficiencia y equidad en la asignación de recursos a una economía, que apuntan a suministrar bienes y servicios públicos, tales como infraestructuras (carreteras, puentes y otros) y servicios sociales (educación, salud, entre otros). Sin embargo, la mayoría de esos "bienes y servicios" se suministran a nivel local (Tiebout, 1956a) e inciden principalmente en las personas residentes en las áreas de suministro. De este modo, la diferencia entre los bienes y servicios públicos que afecta a los residentes de los territorios o unidades espaciales de un país, y que la literatura llama "bienes y servicios públicos locales", es otro aspecto que no se toma explícitamente en cuenta en las definiciones y las teorías de desarrollo económico nacional.

La tercera característica del DEL (a diferencia del DEN) es que en él sí se considera el papel y la participación de los residentes locales (los agentes económicos, políticos y sociales y los ciudadanos) en el proceso. Por una parte, los residentes locales exigen al gobierno (a todos los niveles) bienes y servicios que sirvan para apoyar actividades empresariales y reducir las desigualdades de ingresos en las áreas locales. Por otra, mediante las actividades y la participación de los agentes específicos, junto con las alianzas y asociaciones entre agentes de las áreas locales, también pueden contribuir a aumentar la provisión de bienes y servicios públicos, estimular el crecimiento económico local e influir en las políticas económicas tanto a nivel local como nacional (OCDE, 2007).

El cuarto aspecto que distingue al DEL del DEN es el enfoque multidisciplinario del primero. A fin de abordar la dimensión de la localización geográfica o la dimensión espacial del proceso de DEL, las herramientas clave para analizar este proceso provienen de los campos de la economía regional, urbana, rural y geográfica. El enfoque de las finanzas públicas es el que se emplea para analizar la provisión de bienes públicos locales; asimismo, las herramientas tomadas de la economía política, la sociología y la psicología son necesarias para comprender las acciones y las intervenciones de los agentes en el proceso de DEL. En las siguientes secciones se trata cada uno de los cuatro aspectos mencionados.

\section{III}

\section{Las teorías económicas de localización geográficas y regionales como insumos de las teorías de DEL}

El punto de partida para comprender la relevancia que tienen para el DEL las teorías económicas de localización geográficas y regionales es la teoría de la base económica perteneciente al área de la economía regional. Andrews (1953) define la "base económica" como el conjunto de actividades de una "región" (un área geográfica local o una unidad espacial concreta) que "exporta" bienes y servicios a lugares que se encuentran fuera de sus límites económicos o que vende sus bienes y servicios a compradores que no son de la región. A partir de esta definición, en la teoría de la base económica regional se presupone que el crecimiento económico de la "región" es impulsado por el crecimiento de las "actividades de exportación" de la base económica (Sirkin, 1959, Tiebout, 1956b; North, 1955). ${ }^{4}$

\footnotetext{
${ }^{4}$ Las actividades de la base económica o industrias básicas de una "región" constituyen el conjunto de "artículos o industrias o mate-
}

En las teorías DEL, el conjunto de factores que determinan la localización, las actividades y el crecimiento económico de la base económica de una región ${ }^{5}$

rias primas de exportación". Las actividades que no pertenecen a la base económica, es decir, las industrias no básicas de una "región" las componen las "industrias subsidiarias", formadas o derivadas de las actividades de la base económica. Su demanda es determinada localmente por los residentes de la "región" (North, 1955).

${ }^{5}$ Considerando la demanda del mercado, su distribución geográfica y las dotaciones de recursos locales como elementos exógenos, en las teorías de la localización (Weber, 1957; Isard, 1956 y Von Thünen, 1826) y del lugar central (Christaller, 1966) se introdujeron los costos del transporte y la distancia a los mercados como factores que explican la distribución fija y espacial de las actividades de la región (básicas y no básicas). Por su parte, Marshall (1890) introdujo el concepto de "economías externas o espaciales", vinculadas a la proximidad de los actores económicos dentro de una localización concreta. Tales economías surgen de tres clases de ventajas de los costos y de la localización de los mercados: la creación de empleo y la capacidad de absorber a trabajadores con habilidades especializadas 
proviene de las áreas de la economía de localización y la geografía económica (tanto de la tradicional como de la nueva). Entre otros factores figuran: los factores externos no ubicados en la "región"; las dotaciones de recursos locales (humanos, naturales y capital) e infraestructura física; la distancia a los mercados (de donde procede la demanda de exportación de la base económica); los costos del transporte (que afectan a las características de producción de los bienes y servicios producidos en la base económica y también a su distribución espacial); las aglomeraciones espaciales (o externas) y las economías de escala externas.

En una serie de artículos (Fujita y Krugman, 1995; Fujita y Mori, 1997; Fujita, Krugam y Mori, 1999; Stahl, 1987), las nuevas teorías de la geografía económica iniciadas por Krugman (1991) y Fujita (1988) han introducido todos estos conceptos de manera formal (utilizando las decisiones racionales y optimizadoras de los agentes, las interacciones entre agentes, la mano de obra calificada y la movilidad del capital en un marco de equilibrio general), teniendo en cuenta la localización endógena de las actividades manufactureras y agrícolas y explicando la aglomeración de actividades alrededor de la ciudad y el crecimiento económico de las regiones. La aglomeración de actividades (del consumidor y del productor) en una unidad espacial se presenta formalmente como el resultado de dos fuerzas: las fuerzas centrípetas o de empuje y las fuerzas centrífugas de dispersión o expulsión. Las primeras se derivan de las

que se sienten atraídos a las áreas locales y forman una reserva de trabajadores; la creación de demanda de insumos (especializadas y complementarias) cuya producción es rentable dada la proximidad de los mercados de producción, y la generación de efectos indirectos tecnológicos mediante el intercambio de información y métodos de producción por parte de empresas ubicadas en la misma unidad espacial. Weber (1957) introdujo el concepto de "economías de aglomeración", que surgen de los ahorros en la transacción resultantes de la proximidad de las empresas dentro de una localización específica; y Marshall (1890) también introdujo las "economías de escala externas", definidas como los ahorros en los costos que acumula una empresa a causa del tamaño o del crecimiento de la producción de toda la industria. Dichas economías están en oposición directa a las economías de escala internas, fuente de rendimientos crecientes derivados del tamaño de las fábricas. Las economías externas son, en esencia, factores externos espaciales que pueden definirse en general como consecuencias económicas indirectas de la proximidad entre los actores económicos. Pueden ser negativas o positivas, estáticas o dinámicas, pecuniarias o tecnológicas. La economía externa estática es reversible, mientras que las economías externas dinámicas están vinculadas al progreso tecnológico, el aumento de la especialización y la división del trabajo que acompañan al crecimiento y el desarrollo o los impulsan (Young 1928). Los factores externos pecuniarios se internalizan por medio de mecanismos del mercado; los tecnológicos no, aunque pueden internalizarse por medio de otros mecanismos distintos del mercado. economías espaciales, de aglomeración o externas, de la creación y el desarrollo de eslabonamientos (hacia atrás o hacia delante) o de los efectos del tamaño del mercado. El segundo grupo de fuerzas lo generan la inmovilidad de factores, como la tierra o los trabajadores (las retribuciones de cada factor disminuyen a medida que aumenta la distancia a las actividades aglomeradas), una competencia feroz y deseconomías externas puras (Krugman, 1999, y Fujita y Thisse, 1996).

En la literatura sobre DEL se han formulado diversos mecanismos mediante los cuales la aglomeración de actividades económicas (básicas y no básicas) genera crecimiento y desarrollo económicos para la región local o la unidad espacial en su conjunto. El primero de ellos es el mecanismo multiplicador de ingresos/empleo (Sirkin, 1959), gracias al cual incrementos de los ingresos y empleo en las actividades de la base económica aumentarán la demanda de bienes y de mano de obra en las actividades no básicas. Un segundo mecanismo, inspirado en los modelos de "productos principales y salida de excedentes" (Findlay y Lundahl, 1994), opera mediante los eslabonamientos intersectoriales hacia atrás y hacia delante generados por las actividades de la base económica cuando en la región hay recursos que no se utilizan. Los modelos de crecimiento multisectorial y multirregional se han basado en esos dos mecanismos de los modelos DEL de la base económica (Loveridge, 2004; Nijkamp, Rietveld y Snickars, 1987).

El tercer mecanismo es el de las "economías externas y de aglomeración" como fuentes de las fuerzas centrípetas y centrífugas de las actividades aglomeradas de una región. En los polos de crecimiento y desarrollo (Perroux, 1950, 1955 y 1988) y las teorías de desarrollo del ciclo de un producto (Vernon, 1966) se formularon las ideas básicas, luego modeladas formalmente con el enfoque de la nueva geografía económica (Fujita y Thisse, 2003; Walz, 1996; Baldwin y Forslid, 2000; Black y Henderson, 1999; Martin, Gianmarco y Ottaviano, 1999 y 2001).

Según Perroux (1950), un espacio económico, conceptualizado como un campo de fuerzas, se compone de centros (o polos) a partir de los cuales salen fuerzas centrífugas y a los cuales llegan fuerzas centrípetas. Como motivo de esa aglomeración, Perroux sostuvo que las empresas dominantes (líderes) son comparativamente eficientes y capaces de hacer un uso eficaz de las innovaciones y aumentar así su producción más que otras empresas. Este efecto se propagaría y la sociedad lo percibiría a través de un proceso multiplicador. Por consiguiente, para que las masas se beneficien debe darse cierto proceso de polarización. Perroux (1955) postuló 
también que el crecimiento económico no aparece en todas partes al mismo tiempo, sino que se manifiesta en puntos o "polos" de crecimiento de intensidad variable y se propaga por diferentes vías de efectos terminales variables para el conjunto de la economía. En consecuencia, un polo de crecimiento es una "suma de industrias propulsoras" conectadas con el entorno de la periferia y constituye un "conjunto capaz de inducir el crecimiento (definido como aumento duradero de un indicador dimensional) de otro conjunto". Por otra parte, Perroux (1988) añade que el polo de desarrollo es un "conjunto capaz de engendrar estructuras económicas y sociales cuyo efecto es incrementar la complejidad del todo y expandir su rendimiento multidimensional". Sobre la base de las etapas dinámicas de los productos o de los "ciclos de un producto" (Levitt, 1965), ${ }^{6}$ y de un modo similar al papel destacado de las empresas líderes y eficientes en la creación de polos de crecimiento, Vernon (1966) sostiene que las regiones capaces de producir en las fases de desarrollo de mercado y de crecimiento del producto presentan una expansión económica más rápida. La capacidad de la región para producir en esas dos fases depende, entre otras cosas, del grado de innovación tecnológica de las empresas ubicadas en ella, de la dotación de empresas regionales innovadoras y de los ingresos generados a nivel regional.

En el enfoque de la nueva geografía económica se han formalizado tales ideas: las fuentes de la aglomeración, la tasa de innovación tecnológica (vinculada a la inversión en actividades de investigación y desarro1lo) y las consecuencias indirectas de la tecnología se modelan como los mecanismos clave que producen el crecimiento económico local. Además, y como resultado de los modelos de crecimiento local de la nueva geografía económica, las regiones se dividen en dos grupos: regiones del centro, es decir, las más desarrolladas, y

\footnotetext{
${ }^{6}$ Levitt (1965) distingue cuatro fases: i) la fase de desarrollo del mercado o generación de un producto antes de que haya una demanda comprobada de este y, a menudo, antes de que se haya probado técnicamente en todos sus aspectos. Las ventas son escasas y avanzan lentamente; ii) la fase de crecimiento del mercado: cuando un producto ha sobrevivido a su introducción, la demanda comienza a acelerarse y el tamaño del mercado total se expande con rapidez. Es el momento de auge de cualquier producto; iii) la fase de madurez del mercado, cuando el aumento de las ventas comienza a disminuir y se aproxima al punto en que comenzará el declive inevitable. En las dos últimas fases, los productos considerados actividades de la base económica generan demanda en industrias subsidiarias o actividades no básicas, y se "exportan" a mercados que se encuentran fuera de las unidades espaciales donde se producen, y iv) la fase de declive del mercado, en que el producto empieza a perder atractivo para el consumidor y se inicia el descenso de las ventas. A ella Vernon (1966) la llamó fase de estandarización y comienza en la fase de madurez del mercado.
}

regiones periféricas, las menos desarrolladas. Las regiones centrales producen bienes incluidos en las primeras tres fases del ciclo de un producto, mientras que las regiones periféricas producen en la fase estandarizada. Los conceptos "centro y periferia" aparecieron por primera vez en la obra fundamental de Prebisch (1959).

Un cuarto mecanismo compartido por los modelos de la nueva geografía económica es el de la "causación circular acumulativa", originada por el efecto "cerrojo" de la aglomeración (Fujita y Thisse, 1996; Arthur, 1989). Gracias a este mecanismo, el conjunto de bienes (por lo general diferenciados en finales o intermedios) de las dos primeras fases del ciclo del producto será producido por empresas innovadoras en ubicaciones donde el mercado es relativamente grande y atractivo (medido por el número de trabajadores o consumidores). Sin embargo, el mercado será relativamente grande y atractivo si un número relativamente grande de productores localizan allí su producción. Así, la concentración del sector líder (por lo general, las manufacturas) en una localización determinada se genera y se fortalece mediante el mecanismo de causación circular acumulativa. ${ }^{7} \mathrm{La}$ actividad inicial (normalmente con tecnología de rendimientos crecientes) y su localización, generadoras de dicho mecanismo, tienen su origen en el efecto cerrojo causado por circunstancias (o acontecimientos) casuales o históricas. ${ }^{8}$

Si bien el DEL depende de los factores de localización que generan el desarrollo de la base y de la región económica mediante mecanismos de transmisión entre la base económica y las actividades no básicas, los bienes y servicios públicos y las políticas económicas también son ingredientes clave de la capacidad de desarrollo local.

\footnotetext{
${ }^{7}$ Un tamaño de mercado relativamente grande es atractivo para las empresas a causa de la demanda potencial (un número relativamente grande de consumidores) que puede existir para sus bienes y la disponibilidad de mano de obra (especialmente calificada), pues un alto número de consumidores también significa un alto número de trabajadores. De esta forma, las empresas querrán insumos y mano de obra a través de conexiones hacia atrás. Además, un tamaño de mercado grande se traduce en precios más bajos y salarios reales más altos, lo que puede inducir a los trabajadores a migrar hacia ubicaciones donde se concentra el sector dominante. De ese modo, las empresas aumentarán la oferta y el número de productos (diferenciados) por medio de conexiones hacia delante, y rebajarán los precios en las ubicaciones donde los producen. Según Fujita y Thysse (1996), en virtud de las conexiones hacia atrás y hacia delante del mecanismo de causación circular acumulativa, los rendimientos crecientes a escala al nivel de la empresa se convierten en rendimientos crecientes a escala para el conjunto de la región.

${ }^{8}$ La tecnología de rendimientos crecientes puede conducir a equilibrios múltiples, con condiciones económicas y hechos casuales que determinan qué equilibrio es el que realmente se produce.
} 


\section{IV}

\section{Bienes públicos locales, gobierno local y políticas de desarrollo económico local}

Son varios los temas que se plantean cuando los bienes y servicios públicos locales (BPL) se introducen en el análisis del proceso de DEL; aquí se analizarán tres de ellos. ${ }^{9}$ El primero es el grado de eficiencia del gobierno central (federal) en la provisión de los bienes y servicios públicos. El segundo es el nivel del gobierno local que puede proveerlos con mayor eficiencia. El tercero es el papel económico y social del gobierno local en el proceso de DEL.

En relación con el primero de estos puntos, los estudios pioneros de Oates (1972) y Olson (1969) ofrecieron un punto de partida para el análisis de la teoría de la descentralización o el federalismo fiscal. En Oates (1999 y 2005) y Bardhan (2002), entre otros, pueden leerse estudios recientes de esta corriente de la literatura. Según el teorema de la descentralización de Oates (1972), si las preferencias de los consumidores no son heterogéneas y los BPL producen externalidades a través de las jurisdicciones locales en que se suministran, la solución más eficiente consiste en que el gobierno central proporcione un nivel común de bienes y servicios públicos a todas las localidades. En cambio, cuando las preferencias son heterogéneas y no hay externalidades de los bienes y servicios públicos a través de las jurisdicciones donde se ofrecen, los gobiernos locales son los más eficientes en suministrar los BPL a sus respectivas localidades. En la teoría del federalismo fiscal de primera generación, Oates (2005) preveía un escenario en que los diferentes niveles del gobierno ofrecían bienes públicos, cuyos patrones espaciales de beneficios caían dentro del ámbito geográfico de las jurisdicciones de los respectivos niveles de gobierno. En la terminología

\footnotetext{
${ }^{9}$ Un cuarto tema, surgido en la década de 1990 y analizado parcialmente en el presente artículo, es la gobernabilidad local. En Liou (2007) y Shah y Shah (2006) pueden encontrarse estudios recientes al respecto. Este tema atañe a las diversas clases de marco institucional de gobierno que mejor sirven al desempeño de su papel económico, y trata básicamente de las fallas de las instituciones gubernamentales al intervenir en los mercados. Un quinto tema vinculado a este, aunque no analizado aquí, es la descentralización (en otras palabras, el traspaso de funciones específicas del gobierno central a los gobiernos locales). Rondinelli y Cheema (1983); Litvack, Ahmad y Bird (1999); y Bardhan (2002), entre otros, realizan estudios sobre este tema.
}

de Mancur Olson (1969), esta asignación de bienes y servicios públicos se denomina "mapificación perfecta" o "equivalencia fiscal".

Sobre la base de: i) los trabajos de elección pública y economía política, centrados en los procesos políticos y el comportamiento de los agentes políticos, y ii) la amplia literatura acerca de los problemas de información, en la teoría moderna del federalismo fiscal (o teoría fiscal de segunda generación), resumida en Oates (1999 y 2005), se analizan los mecanismos de distintas instituciones políticas y fiscales en un marco imperfecto de información y control, enfocándose básicamente en los incentivos que esas instituciones incorporan y en el comportamiento al que inducen a los participantes que maximizan sus utilidades (beneficios). En este contexto, la primera interrogante de si las actividades públicas se descentralizan o no, se analizan desde esta nueva perspectiva. Por otra parte, las ineficiencias de la provisión centralizada de los BPL causadas por bienes y servicios uniformes que no reflejan las divergencias en gustos y condiciones locales, y las ineficiencias de la provisión local de estos BPL generadas cuando las externalidades entre jurisdicciones no son internalizadas, se analizan desde una perspectiva algo (si bien no totalmente) distinta.

El trabajo pionero de Tiebout (1956b) es el punto de partida para abordar el segundo tema, estrechamente ligado al primero. Según Tiebout, los niveles de gobierno se relacionan directamente con el conjunto de bienes y servicios públicos que los gobiernos ofrecen dentro de sus jurisdicciones. Este autor demostró que cuando la movilidad de las familias es alta, estas pueden elegir en condiciones óptimas (y de manera eficaz) la jurisdicción de residencia que ofrece el paquete de bienes y servicios públicos más acorde con sus preferencias. A diferencia de esta teoría de asignación no espacial de bienes y servicios públicos, el enfoque geográfico o de localización aplicado por Hochman, Pines y Thysse (1995) a la provisión de bienes y servicios públicos postula que su consumo conlleva costos de transporte. Tales costos aumentan en función de la distancia entre las ubicaciones residenciales y las facilidades públicas donde los bienes y servicios están disponibles. En consecuencia, 
la descentralización no necesita basarse en las clases de bienes y servicios públicos ofrecidos por los gobiernos locales, sino que puede hacerlo en los territorios. Se ha demostrado que la provisión óptima solo puede descentralizarse mediante gobiernos metropolitanos que proveen toda la gama de bienes y servicios públicos a un o a más de un territorio apropiado. ${ }^{10}$

El tercer tema es el de mayor concentración para los profesionales de DEL. El punto de partida es la teoría del papel económico del gobierno, tal como la formularon Musgrave (1959) y Samuelson (1954). Según estos autores, la eficiencia, la equidad y la estabilidad (macroeconómica) son los tres principios básicos del mercado sobre los que tiene que basarse el papel económico del gobierno. Por lo tanto, las fallas o distorsiones del mercado, tales como la existencia de bienes y servicios públicos y bienes meritorios, las externalidades y los monopolios naturales se han considerado tradicionalmente ineficiencias del mercado que el gobierno debe corregir. Las desigualdades en la distribución de los ingresos, surgidas de la asignación de los recursos del mercado, son otro aspecto que demanda que el gobierno intervenga en la economía. Por consiguiente, las distorsiones y desigualdades del mercado que surgen de la distribución jurisdiccional de los recursos pueden ser características territoriales del mercado, y los gobiernos central (o federal) y local comparten el papel de intervenir en la economía a nivel nacional y local. Por otra parte, el objetivo de la estabilidad macroeconómica se deja como dominio exclusivo del gobierno central. ${ }^{11}$

Recientemente, a partir de los aportes de Musgrave y Samuelson, Shah y Shah (2006) han resumido el cambiante papel económico y las responsabilidades de los gobiernos locales. Bajo el modelo tradicional del federalismo fiscal, basado en el enfoque de las fallas del mercado y la provisión de bienes y servicios públicos, Shah y Shah clasifican los distintos gastos, la provisión de bienes y servicios públicos y las responsabilidades del gobierno en materia fiscal, las que asignan a tres niveles: central (o federal), regional (estados o provincias) y local (municipios o áreas metropolitanas).

\footnotetext{
${ }^{10}$ La jurisdicción geográfica del gobierno local metropolitano es un territorio donde la tasa de usuario cobrada a sus residentes, más la correspondiente renta rural total, es igual al costo de proveer todos los bienes y servicios públicos suministrados por el gobierno metropolitano.

${ }^{11}$ Watt (2006) y King (1984), entre otros, sostienen que la estabilización y la redistribución (normalmente por medio de transferencias) son los roles del gobierno central, mientras que el papel del gobierno local consiste en asignar con eficiencia los bienes y servicios públicos locales.
}

Sobre la misma base del papel del gobierno (fallas del mercado y provisión de bienes y servicios públicos), la nueva perspectiva de gestión pública se centra en lo que los gobiernos locales deberían hacer y en cómo deberían hacerlo mejor. Desde esta óptica, el gobierno (central y local) es considerado el "agente" de la población (la que constituye el "principal"); por lo tanto, su responsabilidad consiste en servir al interés público y crear valor público (definido por Moore (1996) y medido como las mejoras en los resultados sociales o la calidad de vida). Por otra parte, en esta perspectiva se sugiere un cambio en la manera en que el gobierno local debería cumplir con su responsabilidad: desde el enfoque de arriba hacia abajo del federalismo fiscal al enfoque de abajo hacia arriba, en que los gobiernos se comportan como administradores que sirven a la población en sus jurisdicciones locales (Shah, 2005 y Caulfield, 2003).

A diferencia de estos dos enfoques, en la perspectiva de la elección pública y de la nueva economía institucional existe interés por los fracasos del gobierno más que por los del mercado. En estos enfoques se proponen distintas maneras de organizar el gobierno a fin de evitar las ineficiencias causadas por sus propios fracasos. Por una parte, en la literatura sobre elección pública se respalda la doctrina del interés propio y se sostiene que los participantes involucrados en la formulación e implementación de las políticas aprovechen las oportunidades y los recursos para fomentar sus propios intereses. En consecuencia, para trabajar al servicio de los intereses de la población los gobiernos locales necesitan tener autonomía plena en gastos e impuestos locales y estar sujetos a la competencia dentro y fuera del gobierno. Si no se dan estos requisitos, es probable que los gobiernos locales sean ineficientes e insensibles a las preferencias de los ciudadanos (Boyne, 1998). Por otra parte, en la nueva economía institucional se postulan diversos órdenes de gobierno (como agentes) para servir a los intereses de los ciudadanos (como principales). En el diseño jurisdiccional se debería asegurar que esos agentes sirvan al interés público minimizando los costos de transacción que recaen sobre los principales (Williamson, 1985; Horn, 1997; Shah, 2005).

$\mathrm{Al}$ igual que los dos enfoques anteriores, la gobernabilidad en forma de red también se interesa por los arreglos institucionales de gobierno - centrándose tanto en los fracasos del mercado como en los de gobierno- y ofrece orientación específica a la hora de tratar los fracasos gubernamentales en una forma jerárquica de gobernabilidad pública y la participación 
del gobierno local a través de una asociación con múltiples organizaciones. Desde esta perspectiva, se ha propuesto un mecanismo de gobernabilidad en red para los gobiernos locales basado en la confianza, lealtad y reciprocidad entre socios y sin salvaguardas institucionales formales. Las redes formadas sobre la base de intereses compartidos (el interés basado en redes) pueden proporcionar una forma de gobierno estable siempre y cuando estén formadas únicamente por socios que contribuyen con recursos importantes y si entre ellos se verifica un equilibrio de poderes. De esa manera, el gobierno local puede tener la oportunidad de desempeñar un papel catalizador en cuanto a facilitar los roles de las redes de intereses y esperanzas con vistas a mejorar los resultados sociales para los residentes locales (Dollery y Wallis, 2001).

Un enfoque más proactivo del papel del gobierno local es formulado en la literatura sobre DEL resumida por Liou (2007); Bartik (1995 y 2003); Bachtler y Yuill (2001); Blair (1999); Blakely y Bradshaw (2002), entre otros. Además de las teorías tradicionales y modernas del papel económico y las responsabilidades del gobierno local resumidas en Shah y Shah (2006), los profesionales de DEL (de los Estados Unidos y Europa) proponen otro papel para el gobierno local, como es el de implementar políticas que fomenten el desarrollo económico local. La justificación de ese papel se basa en aspectos específicos de los modelos teóricos de DEL que pueden relacionarse o que se argumenta que son coherentes con los papeles de eficiencia y equidad de las teorías tradicionales y modernas del gobierno local.

Según Bartik (2003), la política de desarrollo económico del gobierno local se define como una serie de actividades especiales realizadas por el gobierno local para fomentar el desarrollo económico. Las actividades denominadas "programas de desarrollo económico" se dividen en dos categorías: i) otorgamiento de incentivos y asistencia personalizada a empresas de las que se esperan mayores beneficios de desarrollo económico, e ii) iniciativas estratégicas mediante las cuales se modifican políticas fiscales, de gastos y de regulación gubernamental a fin de fomentar el desarrollo económico local.

Blakely y Bradshaw (1999); Blair (1999) y Bachtler y Yuill (2001) distinguen hasta tres "olas" de programas y políticas de DEL aplicadas por profesionales de este campo en los países desarrollados: en la primera, anterior a la década de 1980 y basada en las teorías de la localización del DEL, predominan los programas (de incentivos y subsidios) diseñados específicamente para atraer a empresas de viejas áreas industriales que están libres para desplazarse a regiones en crecimiento; en la segunda, característica de la década de 1980 y basada en las teorías tradicionales y neoclásicas de desarrollo regional, predominan los programas de desarrollo local (por ejemplo, creación de nuevas empresas, aumento del capital de inversión, incubadoras de desarrollo y asistencia técnica) y, en la tercera ola, de la década de 1990 en adelante, basada en las teorías de la competitividad local o territorial y de aglomeraciones productivas (clusters), predominan las políticas encaminadas a ofrecer un entorno empresarial regional apropiado con hincapié en la asociación público-privada, la colaboración y la coordinación.

Según la perspectiva institucional de los modelos de gobernabilidad local, los gobiernos locales no son los agentes "principales" del proceso de DEL; en su lugar, los ciudadanos residentes en áreas locales en su diversa composición y distintos papeles son los agentes "principales". La participación activa de los ciudadanos también es una característica distintiva de las teorías de DEL. En la sección V se analizan el papel y la participación del ciudadano o del agente privado en el proceso de DEL. 


\section{V}

\section{El papel y la participación de los agentes privados locales en el proceso de DEL}

Diversas clases de ciudadanos o agentes privados (empresarios, mujeres, grupos de agentes que representan al capital social y otros) desempeñan múltiples papeles en la literatura sobre DEL y afectan al proceso de desarrollo local a través de una variedad de mecanismos. ${ }^{12}$ "Emprendedorismo" (Bates, 1993; Malecki, 1994), "intraemprendedorismo" (Pinchot III, 1985) o capital empresarial (Audretsch y Keilbach, 2004a) son los nombres que suelen emplearse en la literatura para identificar a uno de los mecanismos más antiguos impulsados por empresarios y administradores a objeto de generar creación de conocimientos e innovación (Schumpeter, 1934) conducentes al crecimiento económico regional/local (Audretsch y Keilbach, 2007, 2005 y 2004b; Lawton, Glasson y Chadwick, 2005). Sin embargo, la innovación no es la única actividad empresarial que afecta al proceso de DEL. En un informe de la Organización de Cooperación y Desarrollo Económicos (OCDE, 2003) se resumen las actividades e interacciones de los empresarios de áreas locales que afectan a su desarrollo económico y a su proceso de crecimiento. Los empresarios sirven como fuentes de inversión, ahorro, creación de empleo, redes y coordinación de agentes, que pueden mejorar la capacidad de desarrollo de las áreas locales.

Otra manera en que un grupo de ciudadanos puede incidir en el proceso de DEL es en forma de capital social (Trigilia, 2001; Putnam, 1993). Aunque el capital social — definido como el nivel de confianza interpersonal, compromiso cívico y capacidad organizacional que predomina en una comunidad o entre un grupo de ciudadanos-es un rasgo de áreas geográficas locales específicas, el concepto fue concebido originalmente como factor de desarrollo económico a nivel nacional (Woolcock y Narayan, 2000; Zabojnik y Francois, 2005).

Además, Durlauf (2002) señala que el capital social también repercute en temas relacionados con la participación política (DiPasquale y Glaeser, 1999), las

\footnotetext{
12 Por ejemplo: i) gobernadores (propietarios, votantes, contribuyentes, miembros de la comunidad); ii) productores de actividad (por ejemplo, proveedores de servicios, coproductores, grupos de apoyo mutuo que obligan a otros a actuar), y iii) consumidores (clientes y beneficiarios) (Moore, 1996).
}

trampas del desarrollo (Woolcock, 1998), la formación de capital humano (Coleman, 1988) y la eficiencia del sistema judicial (La Porta y otros, 1997). En el primer caso, la inversión de los ciudadanos en capital social podría incluir la membrecía en una organización que podría traducirse en mejoras de la coordinación y acciones políticas dentro de una comunidad. En el segundo caso, la falta de confianza entre los ciudadanos (o de capital social) de una comunidad puede redundar en que persistan trampas del desarrollo. En el tercer caso, las dimensiones de confianza y de coordinación del capital social pueden mejorar los canales de información, las habilidades de comunicación y la creación, el traspaso y los flujos de conocimiento que conducen a la formación de capital humano. Por último, la eficiencia del sistema judicial puede afectar al nivel de confianza entre las personas y así fomentar o desalentar la formación de capital social.

Las mujeres forman otro grupo de ciudadanos cuya participación se ha estudiado recientemente en la literatura sobre DEL (Blumenberg, 1998). Más allá de los temas relativos a la desigualdad de género y la exclusión social en el proceso de desarrollo (Weinberger y Jütting, 2001; Blumenberg, 1998; Elson, 1998), los temas de género y el papel de las mujeres en la familia también se han vinculado a otros aspectos del desarrollo social (tal como se define en Mokate, 2004), por ejemplo: la pobreza, las tasas de fertilidad, la formación de capital humano, la nutrición de la familia y las tasas de mortalidad infantil y materna (Elson, 1998). En lo concerniente al papel de las mujeres en el crecimiento y desarrollo económicos a nivel local, en la literatura sobre DEL se enfatiza en tres papeles: el de empresarias, el de innovadoras (en particular en la venta al por menor y las industrias de servicios) y el de formadoras de capital social (Forsyth, 2000; Molyneux, 2002).

La participación ciudadana también puede influir en el proceso de DEL a través de las "asociaciones" (la cooperación, la colaboración, la coordinación o la asociación) locales entre dos o más grupos de agentes (incluidas las instituciones, las organizaciones comunitarias o privadas y las entidades gubernamentales). Estos comparten objetivos de desarrollo comunes 
basados en la localización y operan dentro de relaciones sociales, culturales, económicas y políticas configuradas espacialmente. Las asociaciones locales son entidades territoriales producto de la necesidad (OCDE, 2007). Aparte de los temas atinentes a las definiciones y a las formas de asociación —como los abastecedores verticales o las asociaciones de compradores, las asociaciones gubernamentales horizontales y laterales, y las asociaciones público-privadas, tal como aparecen en la lista de Camarero, Hernández y San Martín, 2008_-, es mediante sus rasgos de capital social y gobernabilidad local que las asociaciones pueden influir en el proceso de DEL (OCDE, 2007).

El elemento "confianza" del capital social puede mejorar los flujos de conocimiento entre los miembros de las asociaciones locales (Jones, Kashlak y Jones, 2004) y superar las fallas del mercado surgidas de las actividades del mercado derivadas de los objetivos de desarrollo y programas de las asociaciones (para la provisión de infraestructura pública). A su vez, el rasgo de la gobernabilidad local puede permitir superar los fracasos del gobierno derivados del proceso de consecución de los objetivos de desarrollo (proporcionando así estabilidad en un entorno turbulento provocado por los cambios económicos, sociales y políticos, y mejorando la eficiencia del mercado mediante el control y la asignación adecuados de recursos y responsabilidades entre los participantes en las asociaciones, tal como se sugiere en Walsh y Meldon (2004) y en OCDE (2001)).

La participación activa de estos y muchos otros grupos de agentes y entidades — como los grupos económicos o de interés estudiados por Gray y Lowery (1988) y los agentes de desarrollo local estudiados por Laukkanen y Niittykangas (2003) — es considerada por los profesionales como parte de cualquier estrategia de planificación encaminada a fomentar el desarrollo económico local (Walsh y Meldon, 2004).

\section{VI \\ El enfoque multidisciplinario de las teorías modernas de DEL}

El cuarto rasgo distintivo de las teorías de DEL es su enfoque multidisciplinario del proceso de desarrollo económico local. Como se ha demostrado en las secciones anteriores, las teorías de DEL reciben insumos de diversas disciplinas (como la teoría espacial y la de localización, la teoría de las finanzas públicas y la teoría de la gobernabilidad, entre otras). No obstante, estas se consideran por separado para subrayar los distintos aspectos de la dinámica del desarrollo local. Hasta la década de 1980 hubo entre los profesionales de DEL un consenso sobre los diversos factores que se toman en cuenta en el proceso de DEL (Thompson, 1968). Sin embargo, a principios de la década de 1990 surgió una serie de teorías con un enfoque multidisciplinario, multidimensional y multifactorial del proceso de DEL, pero dentro de un marco unificado. Esas teorías se han relacionado con la primera ola de políticas de DEL y de programas gubernamentales, y ponen de relieve las interacciones simultáneas de diversos factores a fin de alcanzar los objetivos de desarrollo local. Dos de las teorías "modernas" más estudiadas en la literatura sobre DEL son el enfoque de la competitividad regional/local, o de aglomeraciones productivas (clusters), desarrollado por Porter (1991) y estudiado por Budd y Hirmis (2004), entre otros; y el enfoque de desarrollo mediante clusters (Raines, 2003; Rocha, 2004; Enright, 1996).

Dejando de lado los problemas que implica definir tanto el concepto de competitividad (Lall, 2001) como el de clusters (Martin y Sunley, 2003), el enfoque de Porter se basa en su diamante de la competitividad, que contiene las cuatro fuentes de las ventajas competitivas o de la productividad de las economías nacionales o regionales. Esas fuentes son: las condiciones de la demanda; las condiciones de los factores (o insumos); la estrategia, la estructura y la rivalidad de las empresas; y las industrias relacionadas y de apoyo. Según Porter (2000), las condiciones de la demanda local están mayormente supeditadas al hecho de que las empresas puedan y estén dispuestas a evolucionar de productos y servicios imitativos y de baja calidad a una competencia sobre la base de la diferenciación. En las economías de baja productividad, la mirada se dirige sobre todo a los mercados externos. El fomento precisa del desarrollo de mercados locales más exigentes. La presencia o aparición 
de clientes locales exigentes y experimentados ejerce presión en favor del mejoramiento de las empresas y permite una comprensión de las necesidades actuales y futuras que difícilmente se obtiene en mercados extranjeros. La demanda local también puede hacer visibles segmentos del mercado en los que las empresas pueden diferenciarse. En una economía global, la calidad de la demanda local pesa mucho más que su tamaño.

Porter (2000) sostiene que los "insumos" abarcan desde los activos tangibles, como la infraestructura física, hasta la información, el sistema jurídico y los institutos académicos de investigación, a los que todas las empresas recurren para competir. Si el objetivo es aumentar la productividad, los insumos deben mejorar en eficiencia, calidad y (a la larga) en especialización para los clusters. Los factores especializados, en particular aquellos que forman parte de la innovación y mejoras (de institutos universitarios especializados), no solo se necesitan para alcanzar altos niveles de productividad, sino que también propenden a ser menos negociables o a estar menos disponibles en otros lugares.

Por otra parte, el contexto para la estrategia y la rivalidad de las empresas se refiere a las reglas, los incentivos y las normas que rigen el tipo y la intensidad de la rivalidad local. Las economías de baja productividad se caracterizan por una escasa rivalidad local. La mayor parte de la competencia, si está presente, procede de las importaciones. La rivalidad local, si existe, implica imitación. El precio es la única variable de competencia y las empresas bajan los salarios para competir en los mercados locales y extranjeros. La competencia conlleva una inversión mínima.

El paso a una economía avanzada requiere el desarrollo de una rivalidad local vigorosa, que debe pasar de los salarios bajos a un costo total bajo y para ello es necesario mejorar la eficiencia de las manufacturas y de la entrega de servicios. A la larga, la rivalidad también debe evolucionar a partir de los costos para incluir la diferenciación. La competencia debe pasar de la imitación a la innovación y de una inversión baja a una inversión alta no solo en activos físicos, sino también en activos intangibles (habilidades y tecnología, por ejemplo). Como resulta evidente, las aglomeraciones productivas desempeñan un papel integral en estas transiciones.

Mientras que la naturaleza de la rivalidad en una localización dada acusa una fuerte influencia de muchos aspectos del entorno empresarial (factores disponibles, condiciones de la demanda local), el clima para la inversión y las políticas de competencia definen el contexto. Cuestiones como la estabilidad macroeconómica y política, el sistema fiscal y las políticas del mercado de trabajo afectan a los incentivos para el desarrollo de la fuerza de trabajo, y las normas de propiedad intelectual y su aplicación hacen que las compañías estén menos dispuestas a invertir con vistas a mejorar el equipo de capital, las habilidades y la tecnología. La política antimonopolios, la propiedad gubernamental y las normas de licencias, así como las políticas relativas al comercio, la inversión extranjera y la corrupción, desempeñan un papel fundamental a la hora de definir la intensidad de la rivalidad local.

Por último, las industrias relacionadas y de apoyo se refieren a la presencia o ausencia, a nivel local, de oferentes de materiales, componentes y maquinaria y equipo, además de las industrias afines que apoyan la productividad y la competitividad empresarial local (Porter, 1998). Según Porter (1998), el nivel y la tasa de crecimiento de la productividad en una localización concreta dependen más del modo en que las industrias y las empresas compiten entre sí, que de aquello en lo que compiten. Las fuentes de la competencia definen los factores que influyen en el modo en que las empresas compiten y de esa manera afectan a la productividad y al proceso de DEL en las áreas locales.

Una variante estructural del enfoque de Porter es la competitividad sistémica esbozada en Meyer-Stamer, Altenburg y Hillebrand (1998) y en Meyer-Stamer (2005), en la que al concepto de sistémico se intenta incorporar los determinantes políticos y económicos del desarrollo industrial exitoso. La competitividad sistémica se refiere a una estructura en que el Estado y los actores sociales crean deliberadamente las condiciones para un desarrollo industrial exitoso. En el concepto se distinguen cuatro niveles: el "nivel micro" de la empresa y las redes interempresas; el "nivel meso" de las políticas e instituciones específicas; el "nivel macro" de las condiciones económicas genéricas, y el "nivel meta" de las variables "cualitativas", como las estructuras socioculturales, el orden y la orientación de la economía básica, y la capacidad de los actores sociales para formular estrategias.

A nivel local, un cluster geográfico es el vehículo mediante el cual las áreas geográficas específicas pueden llegar a ser más competitivas y alcanzar la competitividad sistémica para un desarrollo económico e industrial exitoso (Porter, 1996 y 1998). Este cluster es definido como un "grupo geográficamente próximo de compañías interconectadas e instituciones asociadas en un campo particular y vinculadas por características comunes y complementarias. El ámbito geográfico de los clusters puede ir desde una región, un estado o, 
incluso, una ciudad, hasta abarcar países cercanos o vecinos" (Porter, 2000).

A diferencia del enfoque de la competitividad, el enfoque de los clusters se centra en la incidencia de características específicas de estos clusters en el proceso de DEL, vinculadas intrínsecamente a las "economías" y a las propiedades generadas en una localización geográfica. Bajo el primer enfoque, Porter (1991) y Meyer-Stamer, Altenburg y Hillebrand (1998) sostienen que la competitividad y su vehículo de cluster también pueden aplicarse a nivel nacional y no están necesariamente conectados a las propiedades de desarrollo de áreas geográficas específicas. ${ }^{13}$ Además de los factores que determinan la competitividad a nivel local, el enfoque de clusters se centra en las siguientes características de DEL de los clusters localizados en áreas geográficas concretas: conexiones e interdependencia entre empresas y actividades dentro de un espacio dado (Feser, 1998b); factores externos (incluidas las consecuencias indirectas tecnológicas) y las economías de aglomeración que surgen de la localización (Feser, 1998a); la formación de redes sociales distintas al mercado entre agentes dentro del cluster geográfico (Jones, Hesterly y Borgatti, 1997; Powell, 1990); el entorno de innovación (Audretsch, 1998; Audretsch y Feldman, 1996), y los efectos "cerrojo" y de los senderos de dependencia (Kenney y von Burg, 1999; Antonelli, 2000).

Las características del factor de localización y del desarrollo de clusters también se han relacionado con

${ }^{13}$ Enright (1998); Raines (2001) y Camagni (2002) resumen el aspecto geográfico o territorial del concepto de competitividad.

\section{VII}

\section{Conclusiones}

En las tres últimas décadas, la implementación del proceso de descentralización en los países en desarrollo ha generado una demanda de marcos conceptuales que permitan definir los objetivos y papeles apropiados de los agentes públicos y privados en los procesos de DEL. A diferencia de la situación en el mundo industrializado, en la mayoría de los países menos desarrollados el DEL es una disciplina académica prácticamente inexistente. Esta disciplina ofrece cuatro enfoques teóricos para el análisis del proceso de DEL en las economías en desarrollo, que la literatura emergente sobre sistemas de innovación (nacionales y regionales), el aprendizaje y las economías basadas en el conocimiento (Lundvall y Johnson, 1994; Morosini, 2004; Maskell, 2001; Cooke, 2001). En una economía basada en el conocimiento, definida por la OCDE (1995) como una economía que se basa directamente en la producción, la distribución y el uso del conocimiento y la información, los rasgos de la localización y los clusters pueden actuar como vehículos para la creación de conocimiento y el crecimiento económico de áreas locales. Al respecto, Cappellin (2003) plantea que el proceso de creación de conocimiento es interactivo y combinatorio, y que una mayor proximidad geográfica y una mayor proximidad cognitiva facilitan tanto la combinación de piezas complementarias de conocimiento como la interacción entre diversos actores complementarios. Maskell (2001) añade que el cluster se considera la configuración territorial que tiene más posibilidades de mejorar los procesos de aprendizaje. Por último, Leydesdorff (2006) sostiene que la "dinámica de una economía basada en el conocimiento tiene consecuencias importantes para la función de las regiones. Estas pueden servir de incubadoras en donde los procesos de producción, innovación y difusión estén íntimamente conectados. La densidad de las interacciones locales aumenta las posibilidades de "cerrojo" y, en consecuencia, la (co)formación de trayectorias dentro del sistema. La densidad de las interacciones dentro de los clusters productivos y las regiones determina esa capacidad. Por lo tanto, cabe esperar que, en la economía basada en el conocimiento, las regiones metropolitanas ocupen una posición ventajosa".

van mucho más allá de los fundamentos económicos, las instituciones y el enfoque de las fallas del mercado de las teorías de desarrollo económico nacional. Así, los factores de localización, los bienes y servicios públicos locales, la participación activa de diversos agentes privados y el enfoque multidisciplinario de las teorías de DEL pueden servir para dilucidar cuáles son los objetivos necesarios y los roles de los agentes en los procesos de descentralización y desarrollo económico local de los países en desarrollo. 
Aghón, G., F. Alburquerque y P. Cortés (comps.) (2001), Desarrollo económico local y descentralización en América Latina: un análisis comparativo (LC/L.1549), Santiago de Chile, Comisión Económica para América Latina y el Caribe (CEPAL).

Alburquerque, F., J.L. Llorens y J. del Castillo (2002), Estudio de casos de desarrollo económico local en América Latina, Washington, D.C., Banco Interamericano de Desarrollo.

Andrews, R. (1953), "Mechanics of the urban economic base: historical development of the base concept", Land Economics, vol. 29, $\mathrm{N}^{\mathrm{o}} 2$, Wisconsin, The University of Wisconsin Press.

Antonelli, C. (2000), "Path Dependence, Localized Technological Change and the Quest for Dynamic Efficiency”, Turín, Italia, inédito.

Arthur, W.B. (1989), "Competing technologies, increasing returns, and lock-in by historical events", The Economic Journal, vol. 99, $N^{\circ} 394$, Oxford, Royal Economic Society.

Audretsch, D. (1998), "Agglomeration and the location of innovative activity", Oxford Review of Economic Policy, vol. 14, No 2, Oxford, Oxford University Press.

Audretsch, D. y M. Feldman (1996), "R\&D spillovers and the geography of innovation and production", American Economic Review, vol. 86, № 3, Nashville, Tennessee, American Economic Association.

Audretsch, D. y M. Keilbach (2007), "The theory of knowledge spillover entrepreneurship", Journal of Management Studies, vol. 44, $\mathrm{N}^{\mathrm{o}}$ 7, Oxford, Blackwell Publishing.

(2005), "Entrepreneurship capital and regional growth", The Annals of Regional Science, vol. 39, No 3, Heidelberg, Springer.

(2004a), “Does entrepreneurship capital matter?”, Entrepreneurship Theory and Practice, vol. 28, $\mathrm{N}^{\circ}$ 5, Texas, Baylor University.

(2004b), "Entrepreneurship and regional growth: an evolutionary interpretation", Journal of Evolutionary Economics, vol. 14, $\mathrm{N}^{\circ}$ 5, Heidelberg, Springer, diciembre.

(2004c), "Entrepreneurship capital and economic performance", Regional Studies, vol. 38, № 8, Londres, Taylor \& Francis, noviembre.

Bachtler, J. y D. Yuill (2001), "Policies and strategies for regional development: a shift in paradigm?", Regional and Industrial Policy Research Paper, No 16, Glasgow, European Policies Research Centre.

Baldwin, R. y R. Forslid (2000), "The core-periphery model and endogenous growth: stabilizing and destabilizing integration", Economica, vol. 67, No 267, Londres, London School of Economics and Political Science.

Banco Mundial (2010), "Making Local Economic Development Strategies: a Trainer's Manual" [en línea] www.worldbank. org/urban/local/toolkit/.../defining-led.htm

Bardhan, P. (2002), "Decentralization of governance and development", Journal of Economic Perspectives, vol. 16, No 4, Nashville, Tennessee, American Economic Association.

Bartik, T. (2003), "Local economic development policies", Upjohn Institute Staff Working Paper, $\mathrm{N}^{\circ}$ 03-91, Kalamazoo, Michigan, The W.E. Upjohn Institute for Employment Research, enero. (1995), "Economic development strategies", Upjohn Institute Staff Working Paper, $\mathrm{N}^{\circ}$ 95-33, Kalamazoo, Michigan, The W.E. Upjohn Institute for Employment Research, enero.

Bates, T. (1993), "Theories of entrepreneurship", Theories of Local Economic Development: Perspectives from across the disciplines, R. Bingham y R. Mier (comps.), Londres, Sage Publications.

Bingham, R. y R. Mier (1993), Theories of Local Economic Development: Perspectives from Across the Disciplines, Londres, Sage Publications.
Black, D. y V. Henderson (1999), "A theory of urban growth", The Journal of Political Economy, vol. 107, $\mathrm{N}^{\circ}$ 2, Chicago, University of Chicago Press.

Blair, J. (1999), "Local economic development and national growth", Economic Development Review, vol. 16, № 3 .

(1995), Local Economic Development: Analysis and Practice, Thousand Oaks, Sage Publications.

Blakely, E. (2003), "Conceptualizing local economic development: Part 1", Public Finance and Management, vol. 3, $\mathrm{N}^{\mathrm{o}} 2$, Elizabethtown, Southern Public Administration Education Foundation.

Blakely, E. y T. Bradshaw (2002), Planning Local Economic Development: Theory and Practice, Londres, Sage Publications.

(1999), "What are "third-wave" state economic development efforts?: from incentives to industrial policy", Economic Development Quarterly, vol. 13, No 3, Londres, Sage Publications.

Blumenberg, E. (1998), "Gender equity planning: inserting women into local economic development", Journal of Planning Literature, vol. 13, No 2, Londres, Sage Publications, noviembre.

Boyne, G. (1998), Public Choice Theory and Local Government, Houndmills, Macmillan.

Budd, L. y A. Hirmis (2004), "Conceptual framework for regional competitiveness", Regional Studies, vol. 38, No 9, Londres, Taylor \& Francis.

Camarero, C., C. Hernández y S. San Martín (2008), "Developing relationships within the framework of local economic development in Spain", Entrepreneurship \& Regional Development, vol. 20, $\mathrm{N}^{\mathrm{o}}$ 1, Londres, Taylor \& Francis, enero.

Camagni, R. (2002), "On the concept of territorial competitiveness: sound or misleading", Dortmund, Alemania, European Regional Science Association.

Cappellin, R. (2003), "Networks and technological change in regional clusters", Innovation Clusters and Interregional Competition, J. Bröcker, D. Dohse y R. Soltwedel (comps.), Heidelberg, Springer-Verlag.

Caulfield, J. (2003), "Local government reform in comparative perspective", Reshaping Australian Local Government, B. Dollery, N. Marshall y A. Worthington (comps.), Sydney, University of New South Wales Press.

Coleman, J. (1988), "Social capital in the creation of human capital", American Journal of Sociology, vol. 94, Chicago, The University of Chicago Press.

Cooke, P. (2001), "Regional innovations system, clusters and the knowledge economy", Industrial and Corporate Change, vol. 10, No 4, Oxford, Oxford University Press.

Christaller, W. (1966), The Central Places of Southern Germany, Englewood Cliffs, Prentice-Hall.

DiPasquale, D. y E. Glaeser (1999), "Incentives and social capital: do homeowners make better citizens", Journal of Urban Economics, vol. 45, Amsterdam, Elsevier.

Dollery, B. y J. Wallis (2001), The Political Economy of Local Government, Cheltenham, Reino Unido, Edward Elgar.

Durlauf, S. (2002), "On the empirics of social capital", The Economic Journal, vol. 112, No 483, Oxford, Royal Economic Society.

Elson, D. (1998), Gender and Economic Development, Rotterdam, Directorate General for International Cooperation (DGIS)/NEI.

Enright, M. (1998), "The globalization of competition and the localization of competitive advantage: policies toward regional clustering", Working Paper, Hong Kong, Universidad de Hong Kong.

(1996), "Regional clusters and economic development: a research agenda, Business Networks: Prospects for Regional Development, U. Staber, N. Schaefer y B. Sharma (comps.), Berlin, Walter de Gruyter. 
Feser, E. (1998a), "Enterprises, external economies and economic development", Journal of Pla-nning Literature, vol. 12, № 3 , Londres, Sage Publications.

(1998b), "Old and new theories of industrial clusters", European Research in Regional Science, vol. 8, Regional Science Association International.

Findlay, R. y M. Lundahl (1994), "Natural resources, 'vent-forsurplus,' and the staples theory", From Classical Economics to Development Economics, G.M. Meier, Nueva York, St. Martin's Press.

Finot, I. (2001), "Descentralización en América Latina: teoría y práctica”, serie Gestión pública, № 12 (LC/L.1521-P), Santiago de Chile, Comisión Económica para América Latina y el Caribe (CEPAL). Publicación de las Naciones Unidas, $\mathrm{N}^{\circ}$ de venta: S.01.II.G.64.

Forsyth, F. (2000), "Women's enterprise and business development", Local Economy, vol. 15, № 1, Londres, Taylor \& Francis.

Fujita, M. (1988), "A monopolistic competition model of spatial agglomeration: a differentiated product approach", Regional Science and Urban Economics, vol. 18, $\mathrm{N}^{\circ}$ 1, Amsterdam, Elsevier.

Fujita, M. y J. Thisse (2003), "Does geographical agglomeration foster economic growth? And who gains and who loses from it?", The Japanese Economic Review, vol. 54, Hoboken, John Wiley \& Sons.

(1996), "Economics of agglomeration", Journal of the Japanese and International Economies, vol. 10, № 4, Amsterdam, Elsevier.

Fujita, M. y P. Krugman (1995), "When is the economy monocentric?: von Thünen and Chamberlin unified", Regional Science and Urban Economics, vol. 25, $\mathrm{N}^{\circ} 4$, Amsterdam, Elsevier.

Fujita, M., P. Krugman y T. Mori (1999), "On the evolution of hierarchical urban systems", European Economic Review, vol. 43, $\mathrm{N}^{\mathrm{o}}$ 2, Amsterdam, Elsevier.

Fujita, M. y T. Mori (1997), "Structural stability and evolution of urban systems", Regional Science and Urban Economics, vol. 27, $\mathrm{N}^{\mathrm{o}} 4-5$, Amsterdam, Elsevier.

Gray, V. y D. Lowery (1988), "Interest group politics and economic growth in the US States", American Political Science Review, vol. 82, Washington, D.C., American Political Science Association.

Greffe, X. (2004), Local Governance and Partnerships, París, Universidad de París I - Sorbonne.

Hochman, O., D. Pines y J. Thisse (1995), "On the optimal structure of local governments", American Economic Review, vol. 85, № 5, Nashville, Tennessee, American Economic Association.

Horn, M. (1997), The Political Economy of Public Administration, Cambridge, Cambridge University Press.

Isard, W. (1956), Location and Space Economy, Nueva York, John Wiley \& Sons.

Jones, R., R. Kashlak y A. Jones (2004), "Knowledge flows and economic development through microenterprise collaboration", Journal of Entrepreneurship, vol. 7, $\mathrm{N}^{\circ}$ 1, Londres, Sage Publications.

Jones, C., W. Hesterly y S. Borgatti (1997), "A general theory of network governance: exchange conditions and social mechanism", The Academy of Management Review, vol. 22, № 4, Briarcliff Manor, Academy of Management.

Kenney, M. y U. von Burg (1999), "Technology, entrepreneurship and path dependence: industrial clustering in Silicon Valley and route 128", Industrial and Corporate Change, vol. 8, $\mathrm{N}^{\circ} 1$, Oxford, Oxford University Press.

King, D.N. (1984), Fiscal Tiers, Londres, Allen \& Unwin.

Krugman, P. (1999), "The role of geography in development", Proceedings of the World Bank Annual Conference on Development Economics, Washington, D.C., Banco Mundial.

(1991), "Increasing returns and economic geography", Journal of Political Economy, vol. 99, № 3, Chicago, University of Chicago Press.
La Porta, R. y otros (1997), "Trust in large organizations", American Economic Review, vol. 87, № 2, Nashville, Tennessee, American Economic Association.

Lall, S. (2001), "Competitiveness indices and developing countries: an economic evaluation of the global competitiveness report", World Development, vol. 29, № 9, Amsterdam, Elsevier.

Laukkanen, M. y H. Niittykangas (2003), "Local developers as virtual entrepreneurs. Do difficult surroundings need initiating interventions?", Entrepreneurship \& Regional Development, vol. 15, No 4, Londres, Taylor \& Francis, octubre-diciembre.

Lawton, H., J. Glasson y A. Chadwick (2005), "The geography of talent: entrepreneurship and local economic development in Oxfordshire", Entrepreneurship \& Regional Development, vol. 17, $\mathrm{N}^{\circ} 6$, Londres, Taylor \& Francis, noviembre.

Levitt, T. (1965), "Exploit the product life cycle", Harvard Business Review, vol. 43, Boston, Harvard Business Publishing, noviembrediciembre.

Leydesdorff, L. (2006), "While a storm is raging on the open sea: regional development in a knowledge-based economy", The Journal of Technology Transfer, vol. 31, № 1, Nueva York, Springer.

Liou, K. (2007), "Applying good governance concept to promote local economic development: contribution and challenge", International Journal of Economic Development, vol. 9, $\mathrm{N}^{\circ} 1-2$, Elizabethtown, Southern Public Administration Education Foundation, Inc.

Litvack, J., J. Ahmad y R. Bird (1999), Rethinking Decentralization at the World Bank, Washington, D.C., Banco Mundial.

Llisterri, J. (2000), "Competitividad y desarrollo económico local", Documento de discusión, Washington, D.C., Banco Interamericano de Desarrollo (BID), marzo.

Loveridge, S. (2004), "A typology and assessment of multi-sector regional economic impact models", Regional Studies, vol. 30, $\mathrm{N}^{\mathrm{o}}$ 3, Londres, Taylor \& Francis.

Lundvall, B. y B. Johnson (1994), “The learning economy”, Journal of Industry Studies, vol. 1, No 2, Londres, Routledge.

Malecki, E. (1994), "Entrepreneurship in regional and local development", International Regional Science Review, vol. 16, $\mathrm{N}^{\mathrm{o}}$ 1-2, Londres, Sage Publications.

Malizia, E. (1985), Local Economic Development: A Guide to Practice, Nueva York, Praeger.

Marshall, A. (1890), Principles of Economics, Londres, Macmillan and Co. Ltd.

Martin, R. y P. Sunley (2003), "Deconstructing clusters: chaotic concept or policy panacea?", Journal of Economic Geography, vol. 3, $\mathrm{N}^{\mathrm{o}}$ 1, enero, Oxford, Oxford University Press.

Martin, P., I. Gianmarco y P. Ottaviano (2001), "Growth and agglomeration”, International Economic Review, vol. 42, No 4, Filadelfia, Universidad de Pensilvania/Osaka University Institute of Social and Economic Research Association.

(1999), "Growing locations: industry location in a model of endogenous growth", European Economic Review, vol. 43, $\mathrm{N}^{\mathrm{o}} 2$, Amsterdam, Elsevier.

Maskell, P. (2001), "Towards a knowledge based theory of geographical clusters", Industrial and Corporate Change, vol. 10, $\mathrm{N}^{\circ} 4$, Oxford, Oxford University Press.

McGuire, M. y otros (1994), "Building development capacity in nonmetropolitan communities", Public Administration Review, vol. 54, $\mathrm{N}^{\circ}$ 5, Nueva York, John Wiley \& Sons.

Meyer-Stamer, J. (2005), "Systemic competitiveness revisited", Mesopartner WP, Duisburg, Alemania.

Meyer-Stamer, J., T. Altenburg y W. Hillebrand (1998), Building Systemic Competitiveness. Concept and Case Studies from Mexico, Brazil, Paraguay, Korea and Thailand, Berlin, German Development Institute.

Mokate, K, (2004), Women's Participation in Social Development: Experiences from Latin America and the Caribbean, Washington, D.C., Banco Interamericano de Desarrollo. 
Molyneux, M. (2002), "Gender and the silences of social capital, lessons from Latin America", Development and Change, vol. 33, $\mathrm{N}^{\circ}$ 2, Nueva York, John Wiley \& Sons.

Montero, A. y D. Samuels (2004), Decentralization and Democracy in Latin America, Notre Dame, University of Notre Dame Press.

Moore, M. (1996), Creating Public Value, Cambridge, Massachusetts, Harvard University Press.

Morosoni, P. (2004), "Industrial clusters, knowledge integration and performance", World Development, vol. 32, № 2, Amsterdam, Elsevier.

Musgrave, R.A. (1959), The Theory of Public Finance, Nueva York, McGraw-Hill.

Nijkamp, P., P. Rietveld y F. Snickars (1987), "Regional and multiregional economic models: a survey", Handbook of Regional and Urban Economics, P. Nijkamp (comp.), vol., № 1, Amsterdam, North Holland.

North, D. (1955), "Location theory and regional economic growth", The Journal of Political Economy, vol. 63, № 3, Chicago, University of Chicago Press.

Oates, W. (2005), "Toward a second-generation theory of fiscal federalism", International Tax and Public Finance, vol. 12, $\mathrm{N}^{\circ} 4$, Nueva York, Springer.

(1999), "An essay on fiscal federalism", Journal of Economic Literature, vol. 37, $\mathrm{N}^{\circ}$ 3, Nashville, Tennessee, American Economic Association.

(1972), Fiscal Federalism, Nueva York, Harcourt Brace Jovanovich.

ocDE (Organización de Cooperación y Desarrollo Económicos) (2007), Enhancing the Capacity of Partnerships to Influence Policy, París.

(2003), Entrepreneurship and Local Economic Development: Programme and Policy Recommendations, París.

(2002), Redefining Territories: Functional Regions, París, Working Party on Territorial Indicators.

(2001), Local Partnership for Better Governance, París. (1995), The Knowledge Based-Economy, París.

Olson, M. (1969), "The principle of 'fiscal equivalence': the division of responsibilities among different levels of government", American Economic Review, vol. 59, N ${ }^{\circ}$, Nashville, Tennessee, American Economic Association.

Oxhorn, P., J. Tulchin y A. Selee (comps.) (2004), Decentralization, Democratic Governance, and Civil Society in Comparative Perspective: Africa, Asia and Latin America, Baltimore, The Johns Hopkins University Press.

Perroux, F. (1988), "The pole of development's new place in a general theory of economic activity", Regional Economic Development: Essays in Honor of Francois Perroux, B. Higgins y D. Savoie (comps.), Boston, Unwin Hyman.

(1955), "Note sur la notion de pole de croissance", Economie appliquée, $\mathrm{N}^{\circ}$ 8, París, Les Presses de l'ISMEA. Publicado también en I. Livingstone, Development Economics and Policy: Selected Readings, Londres, George Allen \& Unwin, 1979.

(1950), "Economic space: theory and applications", Quarterly Journal of Economics, vol. 64, Cambridge, Massachusetts, The MIT Press.

Pinchot III, G. (1985), Intrapreneuring: Why You Don't Have to Leave the Corporation to Become an Entrepreneur, Nueva York, Harper \& Row.

Porter, M. (2000), "Location, competition and economic development: local clusters in the global economy", Economic Development Quarterly, vol. 14, № 1, Londres, Sage Publications.

(1998), "The Adam Smith address: location, clusters and the new microeconomics of competition", Business Economics, vol. 33, $\mathrm{N}^{\circ}$ 1, Washington, D.C., National Association for Business Economics.

(1996), "Competitive advantage, agglomeration economies and regional policy", International Regional Science Review, vol. 19, $N^{\circ} 1-2$, Londres, Sage Publications.
(1991), La ventaja competitiva de las naciones, Buenos Aires, Vergara.

Powell, W. (1990), "Neither markets nor hierarchy: network forms of organizations", Research in Organizational Behavior, B. Staw y L. Cummings (comps.), Greenwich, JAI Press.

Prebisch, R. (1959), "Commercial policy in the underdeveloped countries", American Economic Review, vol. 49, № 2, Nashville, Tennessee, American Economic Association.

Putnam, R. (1993), "The prosperous community: social capital and economic growth", The American Prospect, $\mathrm{N}^{\circ} 13$.

Raines, P. (2003), Cluster Development and Policy, Burlington, Ashgate Publishing.

(2001), "Local or national competitive advantage?: the tensions in cluster development policy", Regional and Industrial Policy Research Paper, No 43, Glasgow, European Policy Research Center.

Rocha, H. (2004), "Entrepreneurship and development: the role of clusters", Small Business Economics, vol. 23, N ${ }^{\circ} 5$, Nueva York, Springer.

Rondinelli, D.A. y G.S. Cheema (1983), Decentralization and Development: Policy Implementation in Developing Countries, Londres, Sage Publications.

Samuelson, P. (1954), "The pure theory of public expenditure", The Review of Economics and Statistics, vol. 36, № 4, Cambridge, Massachusetts, The MIT Press.

Schumpeter, J. (1934), The Theory of Economic Development: An Inquiry, Profits, Capital, Interest and the Business Cycle, Cambridge, Massachusetts, Harvard University Press.

Shah, A. (2005), "A framework for evaluating alternate institutional arrangements for fiscal equalization transfers", Policy Research Working Paper Series, № 3785, Washington, D.C., Banco Mundial.

Shah, A. y S. Shah (2006), "The new vision of local governance and the evolving roles of local governments", Local Governance in Developing Countries, A. Shah (comp.), Washington, D.C., Banco Mundial.

Sirkin, G. (1959), "The theory of the regional economic base", The Review of Economics and Statistics, vol. 41, No 4, Cambridge, Massachusetts, The MIT Press.

Stahl, K. (1987), "Theories of urban business location", Handbook of Regional and Urban Economics, vol. 2, E. Mills (comp.), Amsterdam, North Holland.

Stren, R. y otros (2002), Decentralization in Global Perspective: A Review of Twenty-Eight Country Experiences, informe preparado para Naciones Unidas-Habitat, Nairoibi, Kenya, octubre.

Tello, M. (2008), Desarrollo económico local, descentralización y clusters: teorías, evidencias y aplicaciones, Lima, Centrum Católica, Pontificia Universidad Católica del Perú.

Thompson, W. (1968), "Internal and external factors in the development of urban economics", Issues in Urban Economics, H. Perloff y L. Wingo Jr. (comps.), Baltimore, Johns Hopkins Press.

Tiebout, C. (1956a), "A pure theory of local expenditures", The Journal of Political Economy, vol. 64, № 5, Chicago, The University of Chicago Press.

(1956b), "Exports and regional economic growth", The Journal of Political Economy, $\mathrm{N}^{\mathrm{o}}$ 64, Chicago, University of Chicago Press.

Trigilia, C. (2001), "Social capital and local development", European Journal of Social Theory, vol. 4, No 4, Londres, Sage Publications.

Vernon, R. (1966), "International investment and international trade in the product cycle", The Quarterly Journal of Economics, vol. 80, $\mathrm{N}^{\circ} 2$, Cambridge, Massachusetts, The MIT Press.

Von Thünen, J. (1826), Isolated State, Nueva York, Pergamon Press.

Walsh, J. y J. Meldon (2004), Partnerships for Effective Local Development, Charleroi, Université Libre de Bruxelles. 
Walz, U. (1996), "Transport costs, intermediate goods and localized growth", Regional Science and Urban Economics, vol. 26, $\mathrm{N}^{\circ}$ 1, Amsterdam, Elsevier.

Watt, P. (2006), "Principles and theories of local government", Economic Affairs, vol. 26, $\mathrm{N}^{\mathrm{o}}$ 1, Oxford, Blackwell Publishing.

Weber, A. (1957), The Theory of Location of Industries, Chicago, The University of Chicago Press

Weinberger, K. y J. Jütting (2001), "Women's participation in local organizations: conditions and constrains", World Development, vol. 29, $\mathrm{N}^{\circ} 8$, Amsterdam, Elsevier.

Williamson, O. (1985), The Economic Institutions of Capitalism, Nueva York, Free Press.
Woolcock, M. (1998), "Social capital and economic development: towards a theoretical synthesis and policy framework", Theory and Society, vol. 27, $\mathrm{N}^{\circ} 2$, Nueva York, Springer

Woolcock, M. y D. Narayan (2000), "Social capital: implications for development theory, research, and policy", The World Bank Research Observer, vol. 15, No 2, Oxford, Oxford University Press, agosto.

Young, A. (1928), "Increasing returns and economic progress", The Economic Journal, vol. 38, Oxford, Royal Economic Society.

Zabojnik, F. y P. Francois (2005), "Trust, social capital, and economic development", Journal of the European Economic Association, vol. 3, No 1, Cambridge, Massachusetts, The MIT Press. 